\section{$\$$ Research Square}

Preprints are preliminary reports that have not undergone peer review.

They should not be considered conclusive, used to inform clinical practice, or referenced by the media as validated information.

\title{
Integrated Blood Pressure-Chemical Sensing \\ Epidermal Patch
}

\section{Juliane Sempionatto}

University of California, San Diego

Muyang Lin

University of California, San Diego

Lu Yin

University of California, San Diego

Ernesto de la Paz

University of California, San Diego

Kexin Pei

University of California, San Diego

Thitaporn Sonsa-ard

University of California, San Diego

Andre de Loyola Silva

University of California, San Diego

Ahmed Khorshed

University of California, San Diego

Fangyu Zhang

University of California, San Diego

Nicholas Tostado

University of California, San Diego

Sheng Xu

Joseph Wang ( $\nabla$ josephwang@ucsd.edu )

University of California, San Diego

\section{Article}

Keywords: wearable sensors, non-invasive electrochemical detection, multiplexed sensing modalities

Posted Date: July 17th, 2020

DOl: https://doi.org/10.21203/rs.3.rs-40455/v1

License: (c) (1) This work is licensed under a Creative Commons Attribution 4.0 International License. Read Full License 
Version of Record: A version of this preprint was published at Nature Biomedical Engineering on February 15th, 2021. See the published version at https://doi.org/10.1038/s41551-021-00685-1. 


\section{Abstract}

The growing demand for tracking the effects of diverse daily activities upon the body physiological response calls for continuous monitoring devices, with integrated hemodynamic and metabolic sensing modalities. This work presents the first demonstration of an integrated wearable sensor that monitors the blood pressure and heart rate via ultrasonic transducers, along with parallel non-invasive electrochemical detection of biomarker levels, such as glucose, lactate, caffeine, and alcohol, in sweat and interstitial fluid. Such simultaneous non-invasive blood-pressure/chemical sensing was implemented by monitoring the dynamic effects of everyday activities, such as exercise and intake of food and drinks, upon the user's physiological states. Leveraging novel material selection and assembly processes, the multiplexed sensing modalities were optimized to ensure reliable sensing without crosstalk between individual sensors, along with mechanical resiliency and flexibility for conformal contact to curved skin surfaces. The simultaneous acoustic and electrochemical sensors were evaluated on multiple human subjects under different stimuli, and the dynamic correlation of the hemodynamic activities and corresponding metabolic variations was monitored and discussed. Such multimodal blood-pressure/chemical wearable sensor offers a collection of previously unavailable information towards enhancing our understanding of the body's response to common activities while holding considerable promise for predicting abnormal cardiac events and improving remote, telemetric, and personalized healthcare medical outcome.

\section{Main}

Intertwined with concepts of telehealth, internet of medical things, and precision medicine, wearable sensors offer attractive features to actively and remotely monitor physiological parameters. Wearable sensors can generate data continuously without causing any discomfort 1,2 or interruptions to daily activity, thus enhancing wearer's self-monitoring compliance and improving patient care quality. ${ }^{2-4}$ The monitoring of physical parameters, such as electrocardiogram (ECG) ${ }^{5-7}$ and blood pressure (BP), ${ }^{8-10}$ and of biochemical parameters, such as glucose, ${ }^{11-15}$ using non-invasive wearable sensors has been reported. Recent efforts have led to the integration of physical and chemical sensors into a single wearable device, such as sensors for ECG with lactate ${ }^{16}$ or glucose ${ }^{17}$ for monitoring athlete's performance, and temperature with metabolites and electrolytes for signal calibration. ${ }^{12,18}$ Yet, to the best of our knowledge, the in-depth study of the correlation of cardiovascular parameters, particularly BP and heart rate (HR), with biomarker levels using an integrated hybrid wearable sensor, remains unexplored.

$\mathrm{BP}$ and HR, two of the most important vital signs, can dynamically and directly reflect the physiological status of the body. These cardiovascular parameters can be affected by fluctuations of various biomarker concentrations originated from activities, such as movement, stress, or intake of food, drinks, and drugs. Multimodal BP-chemical sensing could thus have a tremendous clinical value, especially for people with underlying health conditions, such as the elderlies, obese individuals, diabetic and cardiovascular patients, as their physiological response to normal day-to-day activities might differ from healthy people. Further, the prevention, diagnosis, and treatment of many diseases can greatly benefit 
from the simultaneous monitoring of cardiovascular parameters and biomarker levels. These include acute and deadly septic shock, which commonly involves sudden drops in BP accompanied by rapidly increasing blood lactate levels ${ }^{19}$ and hypo/hyperglycemia-induced hypo/hypertension which increases the risks of stroke, cardiac diseases, retinopathy, and nephropathy in diabetic patients. ${ }^{20-23}$ The recent global pandemic has also highlighted the urgent needs for remote self-monitoring devices, with particular attention to the management of high BP and diabetes, which are the major factors in the deaths of COVID-19 patients. ${ }^{24} \mathrm{~A}$ comprehensive cardiovascular/biomarker self-monitoring platform would enhance users' self-awareness to their health conditions, and alert them and their caregivers to the occurrence of abnormal physiological changes.

Herein, we present for the first time, a conformal, stretchable, and integrated wearable sensor that can simultaneously monitor BP, HR, and levels of glucose, lactate, caffeine, and alcohol, toward dynamic and comprehensive health self-monitoring. We use ultrasonic transducers for monitoring the BP and $\mathrm{HR}$, and electrochemical sensors for measuring the levels of biomarkers. Through strategic material selection, layout design, and fabrication innovation, we integrated rigid and soft sensor components, namely customized piezoelectric lead zirconate titanate (PZT) ultrasound transducers and printed polymer composites via innovative solvent-soldering process, into a single wearable conformal platform with high mechanical resiliency and free of sensor crosstalk. Such rational design overcomes engineering challenges related to the integration of the different sensing modalities and materials to allow real-time monitoring of cardiovascular parameters and biomarker levels, in connection to parallel sampling of the interstitial fluid (ISF) and sweat biofluids. The resulting epidermal hybrid device can emit ultrasonic pulses and sense echoes from arteries, while stimulating sweat and extracting ISF through iontophoresis (IP), allowing simultaneous measurements of $\mathrm{BP}$ and $\mathrm{HR}$, along with multiple biomarkers in these biofluids. We carried out on-body trials with multiple human subjects experiencing diverse activities and stimuli (exercising, having alcohol, food, and caffeine) (Fig. 1d). The correlations between metabolic variations and hemodynamic activities, under these stimuli, were monitored and evaluated. The improved sensor assembly process, leveraging the SEBS-based stretchable materials, allows the fast and reliable fabrication of a stretchable and conformal epidermal sensor for simultaneous acoustic and electrochemical sensing. Such device offers (i) comprehensive tracking of the effect of daily activities and stimuli upon the users' physiological status, and (ii) enables the collection of previously unavailable data towards understanding of the body response to such stimuli, while addressing the critical postpandemic needs for remote telemetric patient monitoring.

The multimodal sensing platform is depicted in Fig. 1a. Styrene-ethylene-butylene-styrene block copolymer (SEBS) was used as the stretchable and conformal substrate to support the electrodes and connections printed with customized inks (Fig. 1e, Supplementary Fig. 1). The stretchable substrate and inks allow the high conformity, flexibility (Fig. 1f-i) and stretchability (Fig. $1 \mathrm{f}$-ii) required for wearable devices. The BP sensor consists of an array of eight piezoelectric transducers, which are aligned with the carotid artery upon applying on the neck to obtain optimal ultrasonic signals (Supplementary Figs. 2-4). During sensing, the piezoelectric transducers were activated with electrical pulses, transmitting 
ultrasound beams to the artery, and the time of flight of the echoes from the anterior and the posterior walls of the artery was analyzed to gauge the dilation and contraction of arteries (Fig. 1c, h). Detailed information regarding the fabrication of the multimodal sensor is discussed in Fig. $2 \mathrm{~m}$ and Supplementary Note 1. The BP sensor characterization is discussed in Supplementary Note 3 and Supplementary Fig. 10. The chemical sensing has been realized through non-invasive sweat stimulation (via transdermal pilocarpine delivery) at the IP anode, alongside with ISF extraction at the IP cathode. (Fig. 1b). Chronoamperometry (CA) was used for electrochemical detection of the hydrogen peroxide product of the glucose oxidase (GOX), lactate oxidase (LOX), and alcohol oxidase (AOx) enzymatic reactions, while differential pulse voltammetry (DPV) was used for the detection of caffeine. Detailed electrode modification and reaction mechanisms are discussed in Supplementary Fig. 5. The analytical performance of each chemical sensor is shown in Fig. 1g, Supplementary Figs. 6-9, and Supplementary Note 2.

\section{Results And Discussions Crosstalk Study}

The performance of the integrated sensor for multiplexed simultaneous sensing requires reliable data generation from the individual sensors, with no crosstalk between the two sensing modalities. Here, the signal crosstalk between the acoustic and electrochemical transducers was prevented by spatially separating both components and using solid-state ultrasound and sensing hydrogel layers (Supplementary Figs. 20 and 21). The device was designed with optimal distance between the individual detection compartments to ensure successful acoustic BP and HR sensing, IP extraction, and electrochemical monitoring. As shown in Fig. 2a and Fig. 2d, the BP transducers were located $1 \mathrm{~cm}$ below the chemical sensors, a distance optimized by assessing the crosstalk between the neighboring sensors. The signal generation of the acoustic sensor relies on high-voltage high-frequency pulses that may induce signal drift in the chemical sensors, while the IP extraction, potentiostatic sensing, and potentialsweep sensing may also induce noises in the acoustic signals. Such crosstalk effect between the electrochemical and BP sensors were evaluated by recording the corresponding signals during on-body operations. The BP signals were acquired while the potentiostatic electrochemical input was turned on and off repeatedly to assess the effect of the electrochemical sensing of the anodic (Fig. 2e and Supplementary Fig. 18) and cathodic (Fig. 2b) sensors on the BP signal. Similarly, the effect of the acoustic sensing on both sides of the electrochemical sensing was examined by recording the amperometric response while turning the acoustic pulses on and off repeatedly every $30 \mathrm{~s}$ (Fig. 2c, f). It is worth noting that without optimization, the electrochemical detection was subject to a strong signal interference due to the potential drift caused by the biased voltage from the acoustic pulses (Supplementary Figs. 19, 20).

Mechanical Performance 
Mechanical stability is another crucial factor that dictates the reliability of skin-worn sensors when tensile deformations are expected. The impedance of the chemical sensor and the contact resistance to the PZT transducers may vary with the strain applied to the soft conformal device leading to changes in the measured signals that affect the reliability of the device. The stability of the PZT contact upon mechanical stress was realized by developing a novel solvent-soldering process based on the fast dissolution and room-temperature curing of SEBS-based materials. During the assembly process, the PZT transducers can be quickly mounted and bonded onto the SEBS substrate and connected to the SEBSbased stretchable silver ink by wetting the electrode surface with toluene (Fig. $2 \mathrm{~m}$ ). The solvent soldered PZT chips can thus be securely bonded to the printed electrodes without delamination during stretching deformations (Fig. 2n), with their assembly efficiency largely improved compared to previous reports. ${ }^{10}$ The effects of stretching on the sensing performance were assessed by stretching tests at $20 \%$ uniaxial strain. The device was stretched repeatedly along the vertical direction (Fig. $2 \mathrm{~g}$ ) and horizontal direction (Fig. 2j). The ultrasonic echo signals, against a two-layered Ecoflex, and the current CA signal, in buffer solution, were recorded after every 200 cycles of stretch at $20 \%$ strain. As shown in Figs. $2 \mathrm{~h}$ and $2 \mathrm{k}$, although the intensity of the acoustic transducer signal decreased slightly with stretching, the temporal relationship between each peak that corresponded to two echoes did not change, and hence the deformations did not affect the recorded waveform. Similarly, the electrochemical sensors did not show any significant current change as the stretching cycle progressed (Figs. 2i, I and Supplementary Figs. 11, 12). The device has also shown good mechanical resilience after transferring it to the body. Figures $20-q$ illustrate the twisting and bending of the sensor on the skin. The SEM images depicting the surfaces structural changes of the printed stretchable silver and carbon traces are displayed in Supplementary Fig. 13, demonstrating that the printed composites are not affected by the mechanical deformation.

\section{Tracking cardiovascular activities and biomarker levels}

The ability of the device to simultaneously monitor dynamic cardiovascular parameters and biomarkers concentrations allows evaluating the effects of common daily activities on individual's physiological status and to continuously collect data about their response to such everyday activity. The levels of lactate, glucose, alcohol, and caffeine in our bodies can fluctuate due to common daily activities, whose impact on our BP also varies based on individual's physical conditions. The simultaneous measurement of biomarkers and BP allows the data collection of individual's responses to such daily activities. The device's ability to track multiple biomarkers while capturing cardiac parameters can further help deconvolute the additive effects of multiple stimuli on physiological parameters, which holds significant implication towards self-monitoring for personalized health management.

Exercise, comprising any action which demands physical efforts, has a major impact on the body physiological response, including changes in lactate levels, HR, and BP. During prolonged exercising, lactate level elevates due to metabolic stress, HR increases to meet the muscle demand for oxygen, while BP surges due to increased availability of vasodilatory mediators such as nitric oxide. ${ }^{25}$ To study these effects, several volunteering subjects were asked to perform stationary cycling in a fixed level for $30 \mathrm{~min}$, 
followed by 20 min resting. BP was recorded while the sweat was stimulated before and after the exercise for the lactate measurements, and the obtained BP and lactate level data were validated by a commercial cuff-style blood pressure monitor and a blood lactate meter. As expected, significant changes in BP and HR were thus observed in Fig. 3a after the exercise, increasing up to $150 \mathrm{mmHg}$ and $98 \mathrm{bpm}$, respectively (Fig. 3a i-ii). Sweat lactate also increased, with low lactate levels recorded in the beginning and increased two-fold after the exercise (Fig. 3a iv-v). The BP and sweat lactate data collected from the device agreed well with the validation methods, as shown on Fig. 3a iii-vi.

As another commonly seen unhealthy stimulus, excessive alcohol consumption, has shown to increase cardiovascular risks via alcohol-induced hypotension and hypertension. ${ }^{26,27}$ Alcohol may have different effects on the BP, depending on the amount and frequency of its consumption and on genetic factors related to resistance or sensitivity to alcohol. ${ }^{28,29} \mathrm{BP}$ variations upon alcohol ingestion are related to the direct vasodilation, surge in cortisol secretion, and reduced insulin sensitivity. ${ }^{30}$ For the sensor experiments focusing on alcohol as the stimulus, the BP and sweat alcohol level were measured before and 20 min after drinking $200 \mathrm{~mL}$ of an alcoholic beverage (19\% vol.) (Fig. 3b). A commercial alcohol breathalyzer was used for correlation with blood alcohol level. As shown in Fig. 3b, the alcohol consumption resulted in an increased HR (from 69 to $85 \mathrm{bpm}$ ) and BP (120 to $136 \mathrm{mmHg}$ ) of the volunteer (Fig. 3b i-ii). These results agree with studies showing that a single alcohol intake by non-heavy drinkers can lead to a temporary BP spike. ${ }^{30}$ It is worth noting that for heavy drinkers, there might be a considerable BP morning surge that greatly increases the risk of stroke. ${ }^{30}$ Simultaneously, the sensor allows reliable detection of sweat alcohol, as this small polar molecule can be found in sweat with a 1:1 correlation to blood (Fig. 3b iv-v). ${ }^{31}$

Metabolites, such as glucose, can also affect the BP waveform by changing the blood viscosity. Blood viscosity increases under conditions of insulin resistance, altering the flux of blood in the capillaries and hence the shape of the BP pulse. ${ }^{32,33}$ Studies have shown that subjects with high blood pressure are prone to significantly higher blood glucose levels. ${ }^{34}$ To test the effect of the rise in glucose upon the BP, healthy non-diabatic subjects were asked to consume a high sugary meal after fasting. The BP and ISF glucose levels were recorded using the device before and $15 \mathrm{~min}$ after consuming the food, with the glucose level validated using a commercial glucometer at both times. As shown in Fig. $3 \mathrm{c}$ i-ii, the sensor evaluation, during the food consumption experiment, resulted in negligible changes in the BP and HR. In contrast, the electrochemical biosensor readily detected changes in the ISF glucose levels after the meal consumption (Fig. $3 \mathrm{c}$ iv-v). This data is within the expectation for the non-diabetic subject, as glucoseinduced BP changes occur only when glucose levels increase significantly to alter the blood pumping through the arteries, which is not common for non-diabetic individuals whose glucose is readily regulated by the responsive release of insulin.

Lastly, caffeine was chosen as another chemical stimuli commonly used in our daily lives. Caffeineintake is known to lead to an increased BP through the inhibition of adenosine receptor and release of stress hormones, such as norepinephrine or cortisol. ${ }^{35}$ These biochemical changes can result in transient 
contractions of the arterial smooth muscle and influence the vascular tone by phosphodiesterase inhibition. ${ }^{36}$ The effect of caffeine on the BP varies, and are shown to be more pronounced in hypertensive subjects. ${ }^{35,36}$ The epidermal BP/caffeine sensor patch was evaluated on subjects with and without caffeine-intake habits, and their BP and sweat caffeine were measured before and $30 \mathrm{~min}$ after consuming a caffeine-rich $(80 \mathrm{mg})$ sugar-free energy drink. The amount of caffeine in sweat was validated through a standard additions voltammetric method, spiking caffeine to a collected sweat sample (Supplementary Fig. 14). As illustrated in Supplementary Fig. 23b, the on-body tests on subject with habitual caffeine-intake showed no significant changes in the BP and HR after consumption of high caffeine doses, reflecting the caffeine tolerance and healthy blood pressure levels of the volunteer. In contrast, the BP variation was more pronounced for the subject with no habitual caffeine intake (Fig. 3d). The caffeine sensor displayed a flat DPV baseline response prior to the caffeine intake, whereas the sweat DPV recorded 30 min after the caffeine intake showed a distinct anodic peak current at $1.2 \mathrm{~V}$, corresponding to the caffeine oxidation (Fig. 3d-iv-v) ${ }^{37,38}$ Current levels before and after the caffeine intake were compared against the results obtained through the standard additions method for caffeine, showing a good correlation between both parameters (Fig. 3d-vi). Note that the in vitro electrochemical characterization of the caffeine sensor in $\mathrm{pH} 4.5$ showed current peaks around $1.1 \mathrm{~V}$ (Supplementary Fig. 9). Such small potential shift is attributed to the difference in sweat $\mathrm{pH}$, which typically fluctuates between 4.5-7.0.

\section{Device Monitoring Multiple Stimuli}

Then we evaluated the device in real-life scenarios, where people usually experience multiple activities that may have synergistic or counteracting effects on the body's physiological response. The use of the device for monitoring cardiovascular parameters along with multiple biomarker levels was evaluated on subjects exposed to multiple stimuli. A common example of counteracting effect to the glucose levels is exercising along with food intake, as glucose can be quickly consumed during exercise to produce energy ${ }^{39}$. Exercise is also expected to increase the BP and lactate level in the subject, as was shown in previous single-stimuli tests. To study this scenario, the subject was asked to consume a sugar-rich meal, followed by exercising on a stationary bike for $30 \mathrm{~min}$, with the ISF glucose, sweat lactate, and BP monitored before and after each step. As shown in Fig. 4a, normal systolic BP level, high glucose levels (>100 mg/dL) and low lactate level were observed before the biking activity. After the exercise, glucose levels decreased, accompanied by a considerable increase in the BP, HR, and lactate level, as predicted from previous tests. Control experiments - performed without any food or exercise - were used to corroborate that the change in signal resulted solely from the increase of lactate and glucose levels (Supplementary Fig. 25). Overall, Fig. 4a illustrates that the new sensor is able to capture the complex processes resulting from the simultaneous food and exercise stimuli, including the digestion of food to produce glucose as the energy reservoir, the glycolysis reaction consuming the glucose and oxygen to release energy, the increased BP and HR compensating for the oxygen depletion, and the lactate generation during the hypoxic condition in exercise. 
The influence of the simultaneous intake of alcohol and glucose on the BP and HR, simulating a typical alcohol consumption during meals, was also studied on volunteering subjects. Based on previous observations, increasing glucose levels are not expected to cause significant changes in the BP of the subjects, whereas an increasing BP is expected after an alcohol intake. Therefore, an additive effect in the rise in $\mathrm{BP}$ and glucose is expected when combining the intake of alcohol and sugary food. Moreover, the digestion of alcoholic drinks, along with the reduced insulin sensitivity caused by alcohol consumption, can further aggravate the increase in the glucose level and the BP. ${ }^{15}$ On the other hand, excessive alcohol intake can lead to severe hypoglycemia and hypotension, even when combined with glucose intake, particularly for insulin-dependent diabetes subjects. ${ }^{40}$ Therefore, the simultaneous monitoring of glucose and $\mathrm{BP}$ is important for distinguishing the case of moderate or excessive drinking and preventing drinking-induced accidents, especially for subjects with underlying health conditions. ${ }^{41,42}$ Sweat alcohol, ISF glucose, and BP signals were recorded in the fasting state, after the alcohol consumption, and after the food intake. As shown on Fig. 4b iv-vi, before any food or alcohol consumptions, blood glucose and alcohol showed a typical non-diabetic fasting state reading of $90 \mathrm{mg} / \mathrm{dL}$ glucose and a $0 \%$ BAC level, whereas increasing BP, glucose, and alcohol signals were observed for $20 \mathrm{~min}$ after the stimuli. The observed increase in BP following the alcohol intake alone was $16 \mathrm{mmHg}$ (Fig. 3b-iii), rising further to $20 \mathrm{mmHg}$ after the concurrent intake of sugary food (Fig. 4b-iii). Such BP variations demonstrate the synergetic effect of combining alcohol and glucose intakes on the BP. Smaller changes in HR were observed following the alcohol and food intakes as compared to the alcohol intake alone, indicating different mechanism for the increased BP. Control experiments carried out without intakes of food or drink were used to corroborate that the observed signal changes were solely due to the increase of alcohol and glucose (Supplementary Fig. 26).

\section{Continuous BP and Biomarker Monitoring}

The ability of the sensor to capture the dynamic biomarker and BP fluctuations while performing physical activity was also demonstrated. Physically active individuals are expected to have lower resting BP, reducing considerably the risk of heart failure events. ${ }^{43,44}$ The lower resting BP can further be reflected in smaller increase in BP during exercising, as physically active individuals signal the body earlier to release nitric oxide (NO) to promote enhanced vasodilation. ${ }^{43}$ Smaller increases in lactate levels are also expected for active individuals compared with non-active ones. ${ }^{45} \mathrm{BP}$ is expected to decrease following intense exercise activity, eventually retuning to its original value, regardless of the fitness level. ${ }^{46}$ Further, studies demonstrated a close relation between the magnitude of the post exercise BP decrease and the lactate levels, showing that elevated blood lactate levels after high intensity exercise promotes larger differences between pre- and post-exercise BP values. ${ }^{47}$ Such complex dynamic processes thus require the hybrid sensor to operate continuously for capturing these real-time fluctuations throughout the activity. Subjects with different fitness levels (physically active and non-active) were asked to perform a 30 min cycling activity at constant intensity while wearing the device, and their BP and sweat lactate levels were monitored continuously until the exercise was stopped. IP was not used for this portion of the study, as sweat was generated spontaneously from the activity. Validation data were also recorded 
before, 10 min into, and after the exercise. As shown in Fig. 5a (for physically active subject) and $\mathbf{5 b}$ (for sedentary subject), a considerably higher sweat lactate level and increased BP values were observed during the exercise for the sedentary subject compared to the active subject. Higher HR, BP, and sweat lactate levels are expected during exercise for the non-active subjects due to the elevated catecholamine levels compared to physically active subjects, leading to differences in BP depending on the fitness levels and cardiovascular system. ${ }^{48}$

\section{Outlook}

The present work reports the first example of a conformal skin-worn device capable of simultaneous monitoring of $\mathrm{BP}, \mathrm{HR}$, and multiple biomarkers. This advance has been realized by elegantly addressing major engineering challenges in integrating rigid ultrasound transducers and soft and stretchable electrochemical sensors into a single flexible and stretchable platform while ensuring mechanical performance and avoiding signal crosstalk. The novel SEBS-based solvent-soldering process has greatly simplified the assembly of a sensor with complex structure while ensuring reliable mechanical behavior and continuous epidermal BP and biomarker signal recordings under different chemical and physical stimuli and activities. Signal crosstalk between the acoustic and electrochemical transducers was prevented by spatially separating both components and using solid-state sensing hydrogel layers. Repeated mechanical deformation tests demonstrated outstanding durability and reliability of the electrochemical and acoustic sensors.

Such simultaneous acoustic and electrochemical sensing offers continuous monitoring of the users' physiological status and its response to multiple everyday activities and stimuli. This multimodal wearable platform has thus been shown useful for correlating common daily activities, such as exercise, drinking, and eating, with changes in BP, HR, and biomarker levels. The encouraging results support the possibility of developing more advanced hybrid wearable sensors that involve complex integration of chemical and physical sensors on a single conformal platform for simultaneously monitoring multiple relevant parameters. Such sophisticated integration of reliable and comprehensive epidermal sensors can only be realized with the judicious material selection, optimized structural engineering, and novel highthroughput fabrication process in mind. While displaying attractive features, there are still opportunities for improving the BP/HR/metabolites patch: (i) The integrated patch relies on the iontophoretic pilocarpine stimulation of sweat, which limits the operational use due to the depletion of pilocarpine. Long term sweat stimulating drugs (e.g., carbachol) can be used to extend the operation period. (ii) Full miniaturization of the device through the development of electronics with integrated ultrasound and potentiostatic capabilities, along with signal processing and wireless transmission functionalities. (iii) Conducting extensive validations involving a large number of subjects with various health conditions, including diabetes and cardiovascular patients. By addressing these opportunities and adding more sensing parameters, we envision a fully integrated multiplexed wearable health monitoring device that offers significant new insights into the health and physiological status of individuals towards the prevention and management of chronic diseases. This device represents an important first step towards 
multimodal wearable sensors that fuse acoustic and electrochemical sensors towards more comprehensive monitoring of human physiology and a successful telehealth transformation. It thus paves the way into a new field of skin-conformal tools capable of providing important, high-quality, and high-density information regarding the status of human health.

\section{Methods}

\section{Materials and reagents}

Chitosan, acetic acid, bovine serum albumin (BSA), L-lactic acid, sodium phosphate monobasic $\left(\mathrm{NaH}_{2} \mathrm{PO}_{4}\right)$, sodium phosphate dibasic $\left(\mathrm{Na}_{2} \mathrm{HPO}_{4}\right), \mathrm{D}(+)$-glucose, glucose oxidase (GOx) from Aspergillus niger type X-S (EC 1.1.3.4), Nafion ${ }^{\circledR}$, agarose, pilocarpine nitrate, Prussian blue (soluble), toluene, ethanol, and silver flakes were obtained from Sigma-Aldrich (St. Louis, MO). Graphite powder was purchased from Acros Organics (USA). Lactate oxidase (LOx) (activity $101 \mathrm{U} \mathrm{mg}^{-1}$ ) was purchased from Toyobo Corp. (Osaka, Japan). SEBS (G1645) was received from Kraton Coporation (Houston TX, USA) while Ecoflex® 00-30 was purchased from Smooth-on Inc. (Easton PA, USA). Super-P carbon black was obtained from MTI Corporation (Richmond, CA, USA). The ultrasound gel pad (AQUAFLEX®) was purchased from Parker Laboratories Inc. (Fairfield, NJ, USA). All reagents were used without further purification.

\section{Sensor Fabrication, Assembly and Electrode Modification}

The screen-printing was carried out using a semi-automatic MMP-SPM printer (Speedline Technologies, Franklin, MA) and custom stainless steel stencils developed using AutoCAD software (Autodesk, San Rafael, CA) and produced by Metal Etch Services (San Marcos, CA), with dimensions of 12 in $\times 12$ in and $125 \mu \mathrm{m}$ thickness. The electrodes were printed layer-by-layer as illustrated in Supplementary Fig. 1. Bulk PZT was used for ultrasound transducers, which were diced (Disco Automatic Dicing Saw DAD3220) into $0.8 \mathrm{~mm}$ by $3 \mathrm{~mm}$ rectangular-shaped pixels and sandwiched by two layers of stretchable silver inks as electrodes. The connection between the transducers and the silver traces was realized by adding a toluene droplet to the printed silver traces and placing the transducers on the softened ink. After attaching the PZT transducers, the screen-printed ground connection was placed on the sensor by dissolving the printed traces in a similar fashion. Details of the transducer assembly process are illustrated in Supplementary Fig. 2. The biosensor electrodes were subsequently modified by drop casting the respective enzymes and polymer layers. Details of the ink formulation, printing and assembling processes, and the individual drop casting protocols for different biosensors are given in Supplementary Note 1.

\section{Sensor In-vitro calibration}

The fabricated sensors, including the lactate, glucose, alcohol and caffeine biosensors, and the PZT acoustic sensors, were calibrated separately in in-vitro settings. The biosensors were calibrated by using $0.1 \mathrm{M}$ PBS ( $\mathrm{pH}$ 7.4) or $0.01 \mathrm{M}$ acetate buffer ( $\mathrm{pH} 4.5)$ with successive spiking of corresponding analytes, and recording the corresponding CA (for lactate, glucose and alcohol) and DPV (for caffeine). Protocols 
of the in-vitro biosensor calibrations are discussed in detail in Supplementary Notes 2. The calibration of the BP waveform is discussed in Supplementary Notes 3.

\section{Sensor Mechanical Tests}

The mechanical testing was conducted via controlled stretching tests. A programmable motorized linear stage (X-LRQ, Zaber Technologies Inc.) was used for stretching the device with controlled strain and speed. One of the edges of the printed device was taped at the fixed end of the stage and the other to the moving end of the stage. The device was stretched at $3 \mathrm{~mm} / \mathrm{s}$ speed to $120 \%$ of its original length and release back to its original size at the same speed. This process was programmed to be repeated 200 times, so that the device could be taken from the stage for measurements before remounting back for subsequent stretching. The process was repeated until 1,000 cycles of stretching were completed (Supplementary Figs. 11 and 12). The resiliency of the sensor was inspected visually by attaching the device to the skin and subjecting it to various deformations (Fig. 2); the corresponding printed surfaces, before and after repeated stretching, were also characterized via SEM imaging (FEI Quanta 250), as shown in Supplementary Fig. 13.

\section{Sensor Crosstalk Tests}

The crosstalk between the acoustic and electrochemical signals was analyzed on-body by monitoring the changes in one signal while the other signal was generated intermittently. For analyzing the co-sensor interference from the CA electrochemical measurement to the BP waveform, the BP signals were recorded continuously for at least $4 \mathrm{~s}$. The CA was performed by applying a constant voltage of $-0.2 \mathrm{~V}$ to the electrochemical sensors. The BP signal was recorded in two stages, when the detection potential was already being applied and subsequently turned off, and when the detention potential was turned on after the BP recording had already started. BP measurements were performed in the same fashion for the anodic and cathodic sensors. For analyzing the crosstalk effect of the acoustic signal generation upon the electrochemical signal acquisition, the CA signal was recorded continuously for $180 \mathrm{~s}$ while the electric pulses for the BP measurements were delivered to the PZT transducer in an off-on-off-on-off-on pattern with a period of $30 \mathrm{~s}$ for each phase. The crosstalk from the differential pulse voltammetry (DPV) to the acoustic signal was evaluated in the same fashion as in the CA tests. The effect of the acoustic signal upon the caffeine sensor was evaluated in two stages, first by recording the DPV signal while the BP recording was being applied, following by terminating the BP signal when the DPV reached the peak potential and by recording the DPV signal prior to initiating the BP acquisition at peak potential. The corresponding data obtained are included in Fig. 2 and Supplementary Fig. 18.

\section{Sensor On-body Test Protocols}

Epidermal evaluation of the device was performed on healthy consenting subjects with no prior history of heart conditions, diabetes, or chronical pain, and in strict compliance with the protocol approved by the Institutional review board (IRB) at the University of California, San Diego. The device was placed on the neck of the volunteers for all on-body evaluations. Prior to every set of measurements using the integrated sensor, the glucose, lactate, alcohol and BP signals were validated with commercial 
glucometer (ACCU-CHEK, USA), blood lactate (NOVA biomedical, USA), breathalyzer (BACtrack S80 Pro) and FDA approved blood pressure cuff (LOVIA, USA), respectively. Caffeine concentrations were estimated by standard addition methodology using the collected sweat (Supplementary Fig. 14). Sweat stimulation and ISF extraction were realized simultaneously by using a $\mu$ Autolab III electrochemical analyzer to apply a current density of $0.3 \mathrm{~mA} \mathrm{~cm}^{-2}$ between the cathode and anode electrodes for 10 min. Prior to sweat generation, a pre-conditioning step was carried out on the skin by applying the same current density using agarose gels in the cathode and anode compartments for $10 \mathrm{~min}$, following by immediately placement of the device with pilocarpine delivery gel on the conditioned area. Before placing the sensor, the skin was thoroughly cleaned with soap and alcohol wipes. The patch was transferred to the skin by using a double-sided clean laser tattoo transfer adhesive (Papilio, TM). Openings were made in the adhesive film to expose the sensors and IP electrodes to the skin. For all measurements, a single device was used for each volunteer to perform the "before" and "after" tests. The device was kept in the volunteer's neck during the entire experiment, unless otherwise specified. Detailed of the hydrogels fabrication and skin transfer processes are illustrated in Supplementary Figs. 15-17 and Video S1.

Unless specified otherwise, the on-body results were acquired using a $\mathrm{CHI} 1230 \mathrm{~A}$ electrochemical analyzer for the biosensors and 5077PR pulser-receiver (Olympus) for the acoustic sensors. Food intake refers to the intake of a portion of sugar-rich food ( $100 \mathrm{~g}$ cheesecake, $350 \mathrm{kcal}, 22 \mathrm{~g}$ sugar). Alcohol intake refers to the intake of a portion of alcohol ( $200 \mathrm{~mL}$ wine, alcohol $19 \%$ vol.). Caffeine intake refers to the intake of a sugar-free caffeinated drink ( $248 \mathrm{~mL}, 80 \mathrm{mg}$ caffeine). Exercise refers to a $30-\mathrm{min}$ exercise session on a stationary bike with constant intensity followed by a 5 -min cooling period.

Exercising: BP and lactate signals were acquired before and after exercising for three healthy volunteers. The device was removed from their skin during the 30 min stationary bike exercise, following a 5 min cooling period, after which the volunteer's neck was cleaned with soap and alcohol pads for replacing the same sensor on the same area.

Alcohol Intake: BP and alcohol levels were measured before and 20 minutes after the alcohol consumption.

Food Intake: BP and ISF glucose signals were acquired in the fasting state (16 hours) for three healthy volunteers and $15 \mathrm{~min}$ after consuming the sugar-rich food.

Caffeine Intake: Subject volunteer's caffeine levels in sweat were monitored before and 30 min after consuming the sugar-free caffeine drink. Prior to the caffeine ingestion, stimulated sweat was collected for the standard addition caffeine determination.

Simultaneous Alcohol and Food Intake: The dual modality of the sensor was tested by combining alcohol and food intakes. BP, ISF glucose and sweat alcohol levels were measured before and after 20 min of the simultaneous consumption of an alcoholic beverage and the sugar rich food. 
Food Intake and Exercising: The dual modality of the sensor was tested toward the monitoring of blood pressure, glucose and lactate levels. The subject was first asked to consume a sugar-rich food. Fifteen min after the food consumption, ISF glucose, sweat lactate and BP were measured. Next, the device was removed form the subject and the volunteer was asked to perform physical exercise on a stationary bicycle for $30 \mathrm{~min}$ followed by cooldown for $5 \mathrm{~min}$; After the cooldown interval the subject's skin was cleaned and the same sensor was used for subsequent measurement of the SIF glucose, sweat lactate and BP levels.

Continuous Lactate and BP Sensing During Exercising: The sensor was further tested by monitoring dynamic changes in BP and sweat lactate while performing continuous physical activity. Subjects with different fitness levels (physically active and non-active) were asked to perform 30 minutes of cycling activity at constant intensity while wearing the sensor. IP was not used for this portion of the study, as sweat was generated spontaneously from the activity. The BP and blood lactate were measured right before the start of the exercise and the initial sweat lactate level was measured 5 min after starting the exercise when sweat was firstly generated. Within $\sim 10$ minutes, BP and blood lactate signal were recorded again. The BP and blood lactate were recorded also upon completion of exercising for validation.

\section{Data Availability}

The data of this study are available from the authors on reasonable request.

\section{Declarations}

\section{Acknowledgements}

This research was supported by the UCSD Center of Wearable Sensors (CWS) and National Institutes of Health (NIH, grant 1R21EB027303-01A1). J.R.S. acknowledge support from CNPq (grant 216981/2014-0). E.P acknowledges support from UC MEXUS-CONACYT collaborative fellowship (2017-2022). A.A. acknowledges support from Fulbright Egyptian Scholar Program (ay2019-2020). T.S. acknowledges the Royal Golden Jubilee Ph.D. scholarship of the Thailand Research Fund. We would like to thank Kraton Corporation for providing the SEBS samples.

\section{Author information}

\section{Affiliations}

Department of Nanoengineering, University of California, San Diego -CA, United States 
Juliane R. Sempionatto, Muyang Lin, Lu Yin, Ernesto de la Paz, Kexin Pei, Thitaporn Sonsa-ard, Andre N. de Loyola Silva, Ahmed A. Khorshed, Fangyu Zhang, Nicholas Tostado, Sheng Xu and Joseph Wang

\section{Corresponding author}

Correspondence to Sheng Xu: shengxu@ucsd.edu and Joseph Wang: josephwang@ucsd.edu

\section{Contribution}

J.R.S., M.L. and L.Y. (Equal contribution) conceived the original project, designed and performed experiments, analyzed data, participated in the figure design and the writing of the manuscript.

E.P. performed experiments, analyzed data, participated in the figure design and the writing of the manuscript.

K.P. and T.S. performed experiments and analyzed data.

A.N.L.S., A.A.K., and F.Z. performed experiments.

N.T. fabricated the devices.

S.X. and J.W. conceived the original project, planned and guided the experiments, analyzed data, participated in the figure design and the writing of the manuscript, and provided guidance and infrastructures for the projects.

\section{Ethics declarations}

Competing interests

The authors declare no competing interests.

\section{Abbreviations}

AA

Ascorbic acid

AC acetaminophen

AL Alcohol

AOx Alcohol oxidase

BAC Blood alcohol concentration 


\begin{tabular}{|c|c|}
\hline BP & Blood pressure \\
\hline CA & Chronoamperometry \\
\hline $\mathrm{CF}$ & Caffeine \\
\hline DPV & Differential pulse voltammetry \\
\hline ECG & Electrocardiogram \\
\hline EEG & Electroencephalogram \\
\hline EKG & Electrocardiogram \\
\hline EMF & Electromotive force \\
\hline GLU & Glucose \\
\hline GOx & Glucose oxidase \\
\hline $\mathrm{HR}$ & Heart rate \\
\hline IP & lontophoresis \\
\hline ISF & Interstitial fluid \\
\hline LA & Lactate \\
\hline LOx & Lactate oxidase \\
\hline NO & Nitric oxide \\
\hline PB & Prussian blue \\
\hline PZT & Piezoelectric lead zirconate titanate \\
\hline RPM & Rotations per minute \\
\hline SEBS & Styrene-ethylene-butylene-styrene block copolymer \\
\hline SEM & Scanning electron microscope \\
\hline UA & Uric acid \\
\hline US & Ultrasound \\
\hline
\end{tabular}

\section{References}


1. Kim, J., Campbell, A. S., de Ávila, B. E.-F. \& Wang, J. Wearable biosensors for healthcare monitoring. Nat. Biotechnol. 37, 389-406 (2019).

2. Yu, Y., Nyein, H. Y. Y., Gao, W. \& Javey, A. Flexible Electrochemical Bioelectronics: The Rise of In Situ Bioanalysis. Adv. Mater. 32, 1902083 (2020).

3. Khan, Y., Ostfeld, A. E., Lochner, C. M., Pierre, A. \& Arias, A. C. Monitoring of Vital Signs with Flexible and Wearable Medical Devices. Adv. Mater. 28, 4373-4395 (2016).

4. Wang, C., Wang, C., Huang, Z. \& Xu, S. Materials and Structures toward Soft Electronics. Adv. Mater. 30, 1801368 (2018).

5. Lee, S. P. et al. Highly flexible, wearable, and disposable cardiac biosensors for remote and ambulatory monitoring. npj Digit. Med. 1, 2 (2018).

6. Lee, Y.-D. \& Chung, W.-Y. Wireless sensor network based wearable smart shirt for ubiquitous health and activity monitoring. Sensors Actuators B Chem. 140, 390-395 (2009).

7. Yapici, M. K., Alkhidir, T., Samad, Y. A. \& Liao, K. Graphene-clad textile electrodes for electrocardiogram monitoring. Sensors Actuators B Chem. 221, 1469-1474 (2015).

8. Luo, N. et al. Flexible Piezoresistive Sensor Patch Enabling Ultralow Power Cuffless Blood Pressure Measurement. Adv. Funct. Mater. 26, 1178-1187 (2016).

9. Dagdeviren, C. et al. Conformable amplified lead zirconate titanate sensors with enhanced piezoelectric response for cutaneous pressure monitoring. Nat. Commun. 5, 4496 (2014).

10. Wang, C. et al. Monitoring of the central blood pressure waveform via a conformal ultrasonic device. Nat. Biomed. Eng. 2, 687-695 (2018).

11. Bandodkar, A. J. et al. Tattoo-based noninvasive glucose monitoring: a proof-of-concept study. Anal. Chem. (2015). doi:10.1021/ac504300n [doi]

12. Lee, H. et al. Wearable/disposable sweat-based glucose monitoring device with multistage transdermal drug delivery module. Sci. Adv. 3, e1601314 (2017).

13. Kim, J., Campbell, A. S. \& Wang, J. Wearable non-invasive epidermal glucose sensors: A review. Talanta 177, 163-170 (2018).

14. Lee, H., Hong, Y. J., Baik, S., Hyeon, T. \& Kim, D. H. Enzyme-Based Glucose Sensor: From Invasive to Wearable Device. Adv. Healthc. Mater. (2018). doi:10.1002/adhm.201701150

15. Kim, J. et al. Simultaneous Monitoring of Sweat and Interstitial Fluid Using a Single Wearable Biosensor Platform. Adv. Sci. (2018). doi:DOI: 10.1002/advs.201800880

16. Imani, S. et al. A wearable chemical-electrophysiological hybrid biosensing system for real-time health and fitness monitoring. Nat. Commun. 7, 11650 (2016).

17. Hong, Y. J. et al. Multifunctional Wearable System that Integrates Sweat-Based Sensing and VitalSign Monitoring to Estimate Pre-/Post-Exercise Glucose Levels. Adv. Funct. Mater. 28, 1805754 (2018).

18. Gao, W. et al. Fully integrated wearable sensor arrays for multiplexed in situ perspiration analysis. Nature 529, 509-514 (2016). 
19. Gomez, H. \& Kellum, J. A. Lactate in Sepsis. JAMA 313, 194 (2015).

20. Zanella, M. T., Kohlmann, O. \& Ribeiro, A. B. Treatment of obesity hypertension and diabetes syndrome. Hypertension (2001). doi:10.1161/01.hyp.38.3.705

21. Cheung, B. M. Y. \& Li, C. Diabetes and Hypertension: Is There a Common Metabolic Pathway? Curr. Atheroscler. Rep. 14, 160-166 (2012).

22. Epstein, M. \& Sowers, J. R. Brief Review Diabetes Mellitus and Hypertension. Hypertension 19, 403406 (1992).

23. Deedwania, P. Hypertension, Dyslipidemia, and Insulin Resistance in Patients With Diabetes Mellitus or the Cardiometabolic Syndrome: Benefits of Vasodilating $\beta$-Blockers. J. Clin. Hypertens. 13, 52-59 (2011).

24. Yang, J. et al. Prevalence of comorbidities and its effects in patients infected with SARS-CoV-2: a systematic review and meta-analysis. Int. J. Infect. Dis. 94, 91-95 (2020).

25. Halliwill, J. R. Mechanisms and Clinical Implications of Post-exercise Hypotension in Humans. Exerc. Sport Sci. Rev. 29, 65-70 (2001).

26. Carter, J. R., Stream, S. F., Durocher, J. J. \& Larson, R. A. Influence of acute alcohol ingestion on sympathetic neural responses to orthostatic stress in humans. Am. J. Physiol. Metab. 300, E771E778 (2011).

27. Roerecke, M. et al. The effect of a reduction in alcohol consumption on blood pressure: a systematic review and meta-analysis. Lancet Public Heal. 2, e108-e120 (2017).

28. Chen, L., Davey Smith, G., Harbord, R. M. \& Lewis, S. J. Alcohol Intake and Blood Pressure: A Systematic Review Implementing a Mendelian Randomization Approach. PLoS Med. 5, e52 (2008).

29. Maheswaran, R., Gill, J. S., Davies, P. \& Beevers, D. G. High blood pressure due to alcohol. A rapidly reversible effect. Hypertension 17, 787-792 (1991).

30. Ohira, T. et al. Effects of habitual alcohol intake on ambulatory blood pressure, heart rate, and its variability among Japanese men. Hypertens. (Dallas, Tex. 1979) 53, 13-9 (2009).

31. Hauke, A. et al. Complete validation of a continuous and blood-correlated sweat biosensing device with integrated sweat stimulation. Lab Chip 18, 3750-3759 (2018).

32. Monte-Moreno, E. Non-invasive estimate of blood glucose and blood pressure from a photoplethysmograph by means of machine learning techniques. Artif. Intell. Med. 53, 127-138 (2011).

33. Çinar, Y. Blood viscosity and blood pressure: role of temperature and hyperglycemia. Am. J. Hypertens. 14, 433-438 (2001).

34. Filipovsky, J. et al. The relationship of blood pressure with glucose, insulin, heart rate, free fatty acids and plasma cortisol levels according to degree of obesity in middle-aged men. J. Hypertens. 14, 229-235 (1996).

35. Mort, J. R. \& Kruse, H. R. Timing of Blood Pressure Measurement Related to Caffeine Consumption. Ann. Pharmacother. 42, 105-110 (2008). 
36. Nurminen, M.-L., Niittynen, L., Korpela, R. \& Vapaatalo, H. Coffee, caffeine and blood pressure: a critical review. Eur. J. Clin. Nutr. 53, 831-839 (1999).

37. Tai, L.-C. et al. Methylxanthine Drug Monitoring with Wearable Sweat Sensors. Adv. Mater. 30, 1707442 (2018).

38. Ciui, B. et al. Chemical Sensing at the Robot Fingertips: Toward Automated Taste Discrimination in Food Samples. ACS Sensors, 3, 2375-2380 (2018).

39. Asano, R. Y. Acute effects of physical exercise in type 2 diabetes: A review. World J. Diabetes 5, 659 (2014).

40. Klaric, D., Klaric, V. Alcohol-Induced Coma, Hypothermia and Hypotension. J. Membr. Sci. Technol. 05, (2015).

41. Wilson, E. \& Waring, W. S. Severe hypotension and hypothermia caused by acute ethanol toxicity. Emerg. Med. J. 24, e7-e7 (2007).

42. Hillbom, M., Saloheimo, P. \& Juvela, S. Alcohol Consumption, Blood Pressure, and the Risk of Stroke. Curr. Hypertens. Rep. 13, 208-213 (2011).

43. Nystoriak, M. A. \& Bhatnagar, A. Cardiovascular Effects and Benefits of Exercise. Frontiers in Cardiovascular Medicine (2018). doi:10.3389/fcvm.2018.00135

44. Fagard, R. H. Effects of exercise, diet and their combination on blood pressure. J. Hum. Hypertens. 19, S20-S24 (2005).

45. Jia, W. et al, Epidermal biofuel cells: energy harvesting from human perspiration, Angew Chemie Int. Ed., 52, 7233-7238(2013).

46. MacDonald, J. R. Potential causes, mechanisms, and implications of post exercise hypotension. J. Hum. Hypertens. 16, 225-236 (2002).

47. Crisafulli, A. et al. Effect of differences in post-exercise lactate accumulation in athletes' haemodynamics. Appl. Physiol. Nutr. Metab. 31, 423-431 (2006).

48. Kokkinos, P. Cardiorespiratory Fitness, Exercise, and Blood Pressure. Hypertension 64, 1160-1164 (2014).

\section{Supplemental Videos}

Supplemental videos were not provided with this version.

\section{Figures}




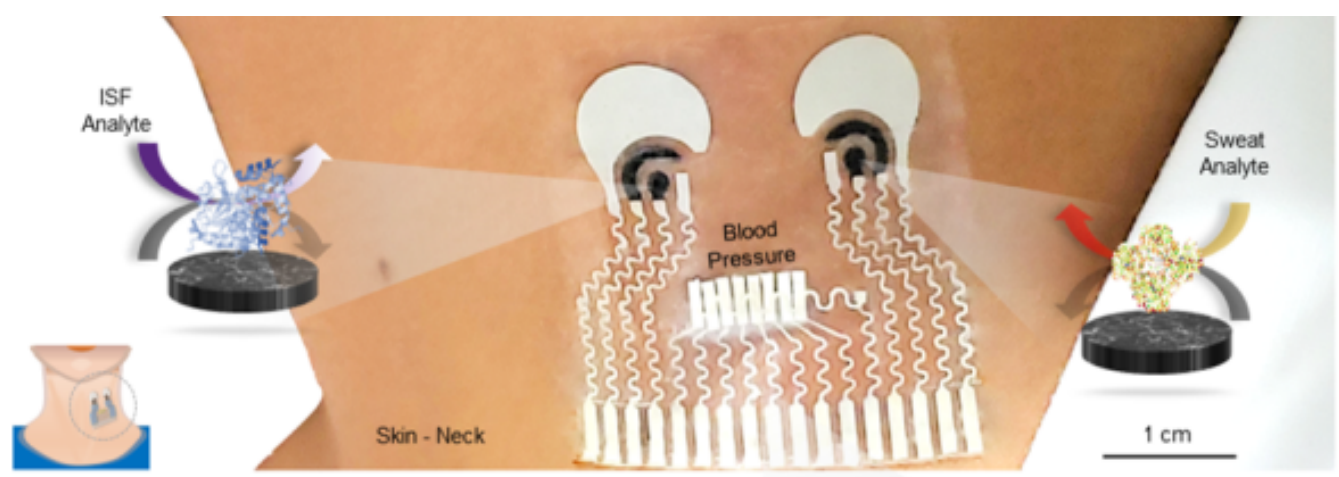

b

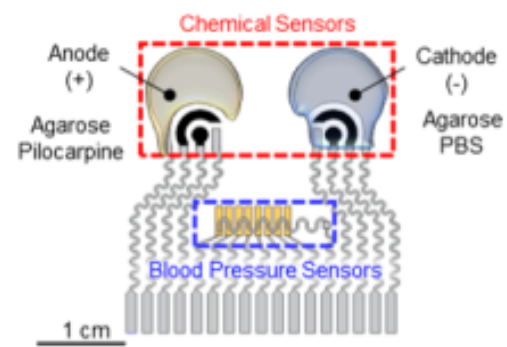

c
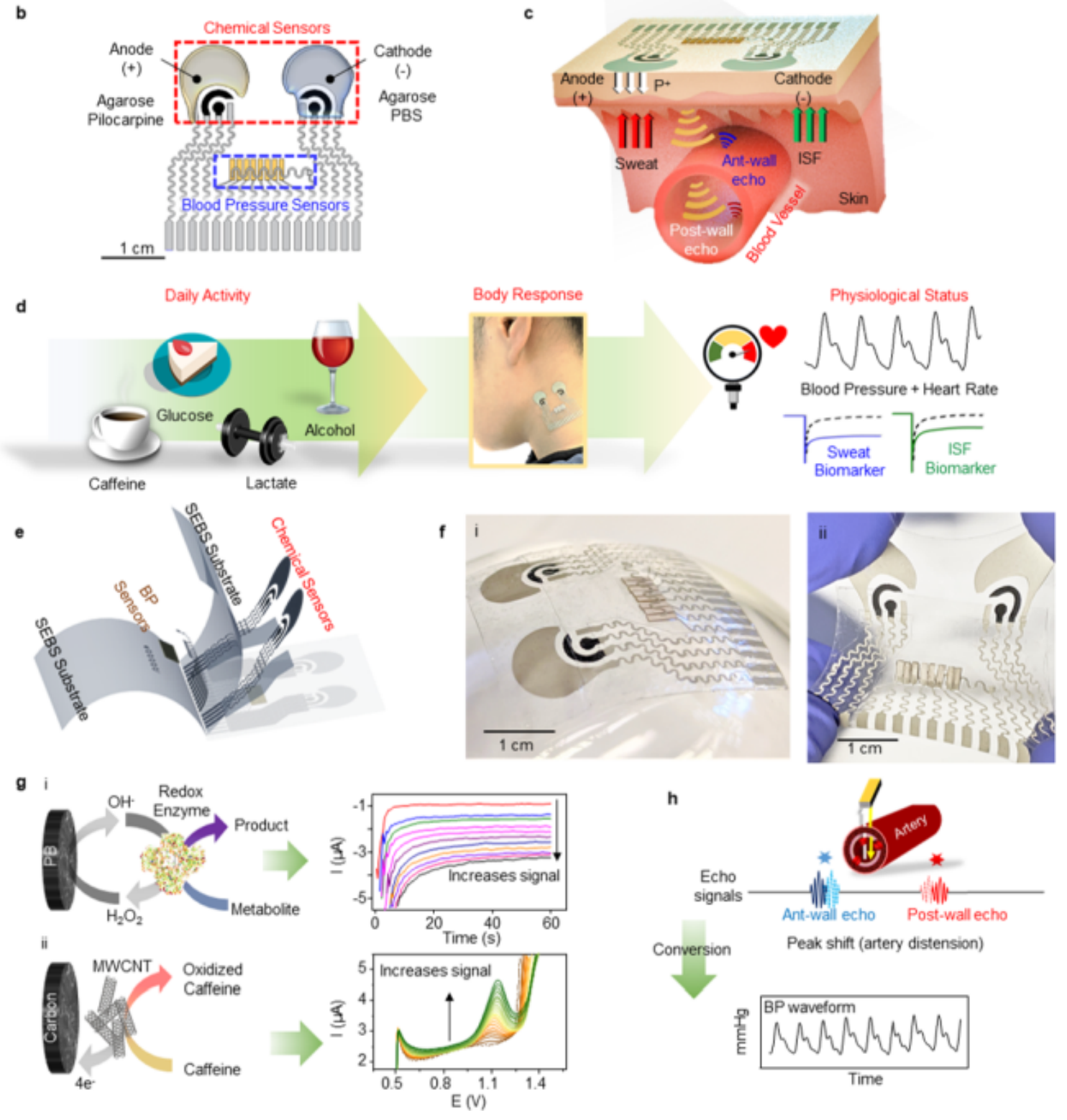

\section{Figure 1}

Design and Mechanism of the stretchable integrated blood pressure-chemical sensing patch. a, A photo image of the sensor on body. b, Illustration of the sensor's acoustic and electrochemical sensing components along with hydrogel for sweat stimulation and ISF extraction. c, Acoustic sensing and iontophoresis mechanism of the integrated sensor. The transducer applies ultrasound pulses which generate echoes from the anterior and posterior walls of the artery. Chemical sensing starts with applying 
an iontophoretic current from a positive terminal (anode + ) to a negative terminal (cathode -) that allows the electro-repulsive delivery of a sweat stimulating molecule P+ (Pilocarpine nitrate). After pilocarpine delivery, stimulated sweat containing biomarkers (such as lactate, caffeine, and alcohol) is collected and quantified in the left side of the device. The iontophoretic current leads to osmotic flow of biomarkers (such as glucose) from the interstitial fluid to the skin surface, allowing its collection and analysis on the right side of the sensor. $d$, Schematic showing the different daily activities inputs performed by an individual and the corresponding biomarkers (alcohol, caffeine, lactate, and glucose) followed by the effect on the individual's system (body response). The inputs are transduced and outputted as BP, HR and electrochemical signals by the device reflecting the body's physiological status. e, Layer-by-layer layout of the integrated sensor. f, Photos of the sensor under i, bending and ii, stretching. g, Mechanisms of the electrochemical sensors. i, amperometric measurements using enzyme-based sensors. The Prussian blue (PB) working electrode was modified either with the LOx, GOx or AOx redox enzymes, allowing the biocatalytic oxidation of lactate, glucose or alcohol molecules to pyruvate, gluconic acid or acetaldehyde (product) respectively, along with the production of hydrogen peroxide. The typical electrochemical reduction of the liberated hydrogen peroxide ( $\mathrm{H} 2 \mathrm{O} 2)$ to hydroxyl ions $(\mathrm{OH}-)$ (Left scheme) was performed in PBS pH 7.4 by applying a potential of $-0.2 \mathrm{~V}$. An increase of negative current is observed by the increase in concentration of chemical analyte (Right scheme). ii, caffeine non-enzymatic measurements. During the sensing process, caffeine was oxidized which resulted in the production of uric acid analog molecules and electrons (Left scheme). A carbon electrode modified with multiwall carbon nanotubes (MWCNT) allowed the pulse-voltammetric detection of caffeine following $30 \mathrm{~s}$ accumulation at $-1.2 \mathrm{~V}$ and scanning between $+0.5 \mathrm{~V}$ and $+1.5 \mathrm{~V}$; Estep: $0.004 \mathrm{~V}$; Epulse: $0.05 \mathrm{~V}$; tpulse: $0.05 \mathrm{~s}$; scan rate: $0.02 \mathrm{~V} / \mathrm{s}$. By increasing the concentration of caffeine, an increasing oxidation signal is observed (Right scheme). h, Signal generation mechanism of the ultrasound transducer. The pulsed ultrasound signal from the transducer is reflected from the anterior and the posterior walls of the artery and collected by the transducer. Signal processing of the ultrasound signal. The time of flight (TOF) of the reflected echo can be converted into BP via established transfer functions. 

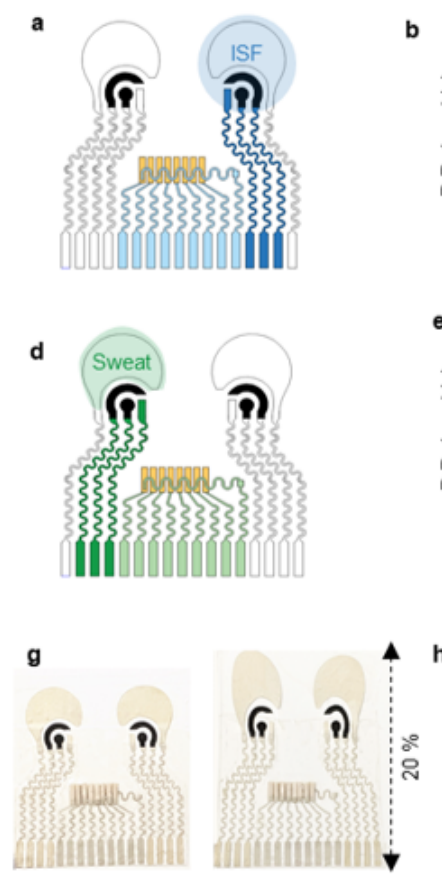

$\overline{0.5} \mathrm{~cm}$
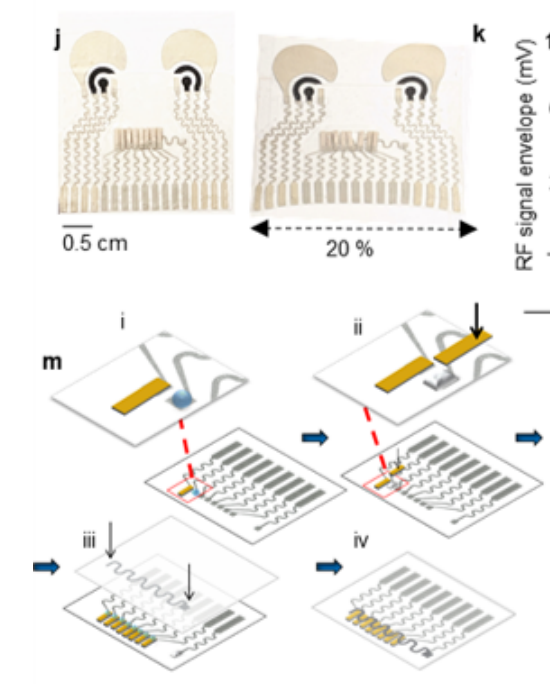

$\circ$

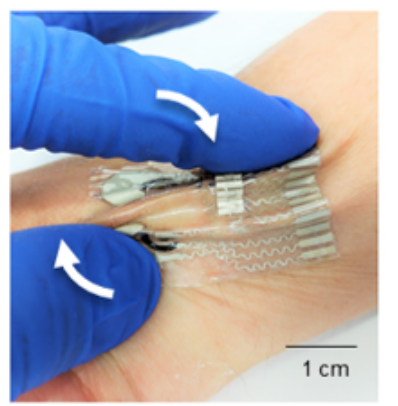

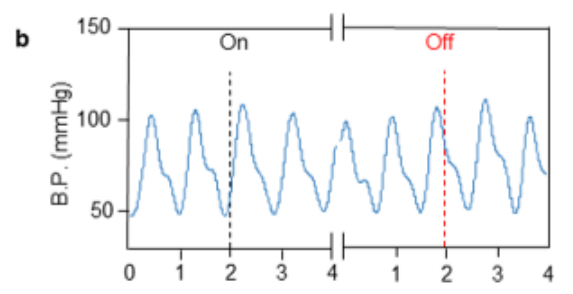
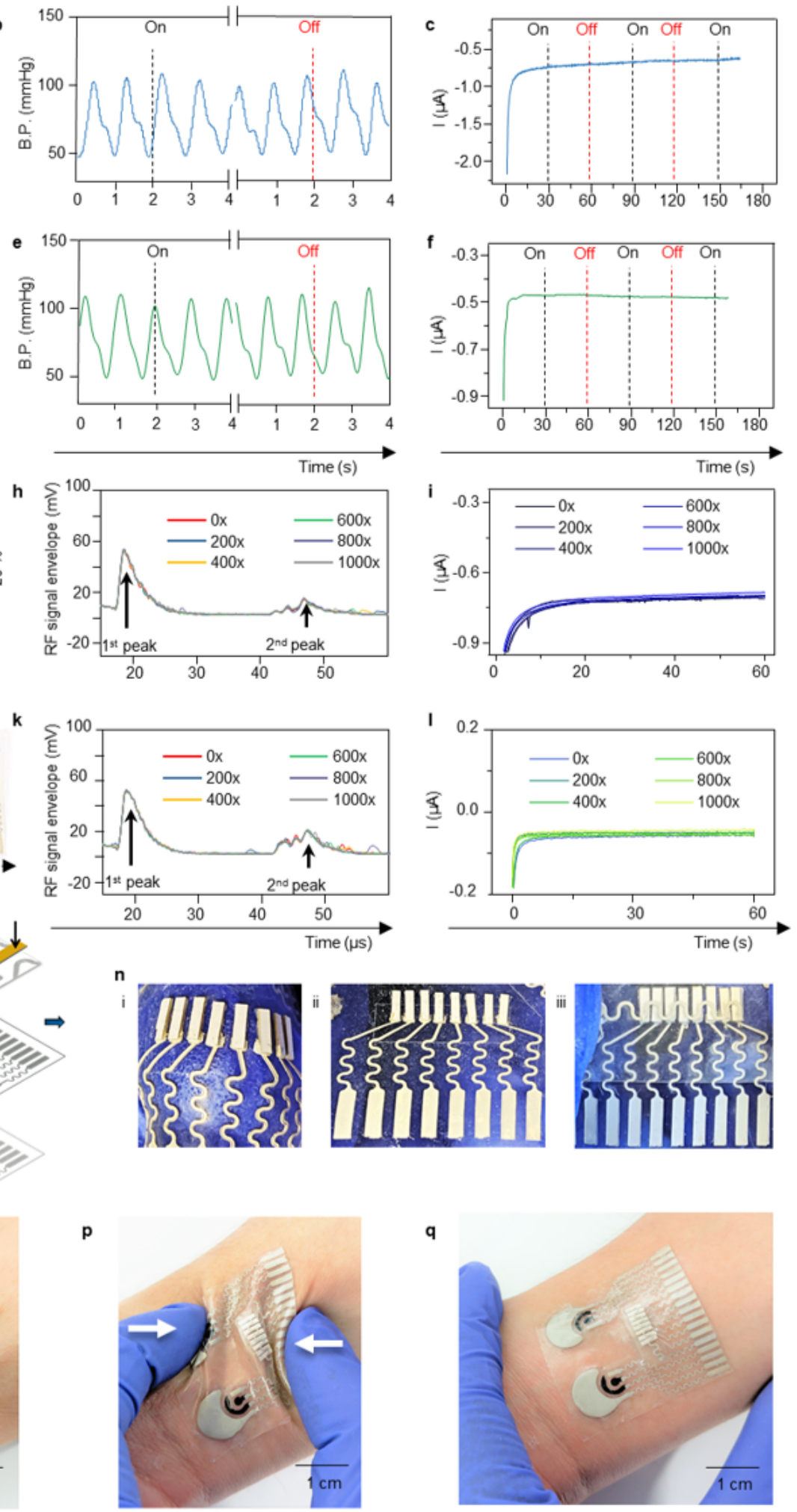

q

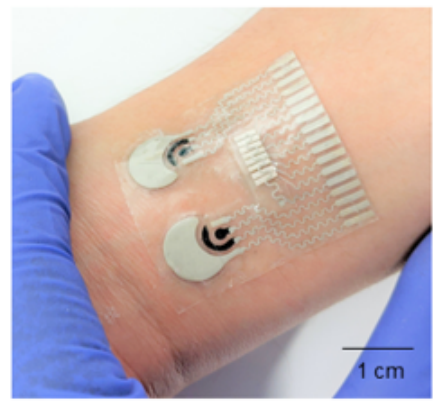

\section{Figure 2}

Characterization of the multimodal wearable sensor. a-c, Signal interference crosstalk studies between the ISF electrochemical and the BP sensors (a), including BP signal recording while applying and removing the CA detection potential (b), and ISF sensor signal recording while start and pausing ultrasound signal generation with $30 \mathrm{~s}$ intervals within $3 \mathrm{~min}$ (c). d-f, Signal interference crosstalk studies between sweat electrochemical sensor and the BP transducer (d), including BP signal recording while 
applying and removing the CA detection potential (e), and sweat sensor signal recording while start and pausing ultrasound signal generation with $30 \mathrm{~s}$ intervals within $3 \mathrm{~min}(\mathrm{f}) . \mathrm{g}$, Photos of the sensor under $20 \%$ vertical strain. h, Envelopes of the raw echo signals before and after every 200 stretching cycles until 1000 cycles. i, Electrochemical response every 200 stretching cycles until 1000 cycles. j, Photos of the sensor under $20 \%$ horizontal strain. $k$, Envelopes of the raw echo signals before and after every 200 stretching cycles until 1000 cycles. I, Electrochemical response every 200 stretching cycles until 1000x m, PZT transfer process. Toluene is drop cast on the electrode pad to dissolve the SEBS trace (i). Softened silver ink ready for bonding with the transducer (ii). Drop cast toluene on transducers for bonding with the ground wire (iii). Binding of the ground layer to the reserved electrode channel (iv). n, Adhesion of the PZT transducers to the substrate. The photo images of the pristine device under indentation (i), during horizontal stretching (ii) and after transferring the ground layer (iii). o-q, Skin conformability and mechanical integrity of the device while twisting (o), bending (p), and after these deformations (q).

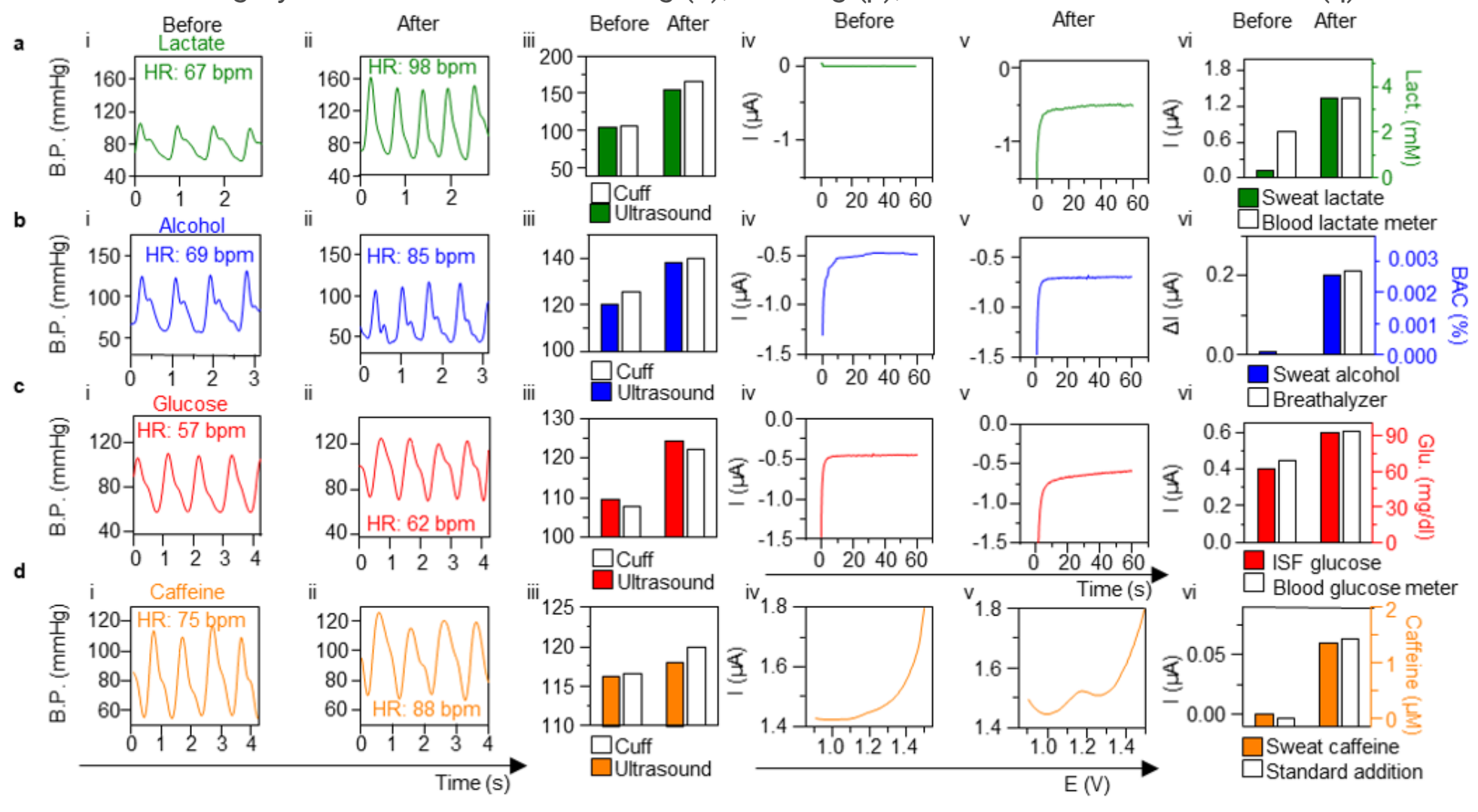

Figure 3

On body evaluation of the hybrid acoustic-electrochemical sensor. a, BP/HR and sweat lactate studies. Signal recording for BP/HR performance before (i) and after (ii) exercise. Bar graphics represent the sensor validation using a commercial cuff (white) and BP readings obtained with ultrasound transducer (green) (iii). Signal recording for sweat lactate before (iv) and after (v) exercise. Bar graphics represent the sensor validation using a commercial blood lactate meter (white) and readings obtained with the electrochemical sensor (green) (vi). b, BP/HR and sweat alcohol studies. Signal recording for BP/HR performance before (i) and after (ii) alcohol intake. Bar graphics represent the sensor validation using a commercial cuff (white) and BP readings obtained with ultrasound transducer (blue) (iii). Signal 
recording for sweat alcohol before (iv) and after (v) alcohol intake. Bar graphics represent the sensor validation using a commercial breathalyzer (white) and readings obtained with the electrochemical sensor (blue) (vi). c, BP/HR and ISF glucose studies. Signal recording for BP/HR performance before (i) and after (ii) food intake. Bar graphics represent the sensor validation using a commercial cuff (white) and BP readings obtained with ultrasound transducer (red) (iii). Signal recording for ISF glucose before (iv) and after (v) food intake. Bar graphics represent the sensor validation using a commercial blood glucometer (white) and readings obtained with the electrochemical sensor (red) (vi). d, BP/HR and sweat caffeine studies. Signal recording for BP/HR performance before (i) and after (ii) caffeine intake. Bar graphics represent the sensor validation using a commercial cuff (white) and BP readings obtained with ultrasound transducer (orange) (iii). Signal recording for sweat caffeine before (iv) and after (v) caffeine intake. Bar graphics represent the sensor validation through standard addition method (white) and readings obtained with the voltammetric sensor (orange) (vi). 


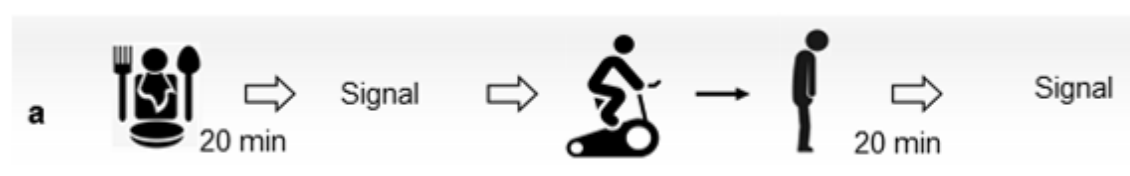
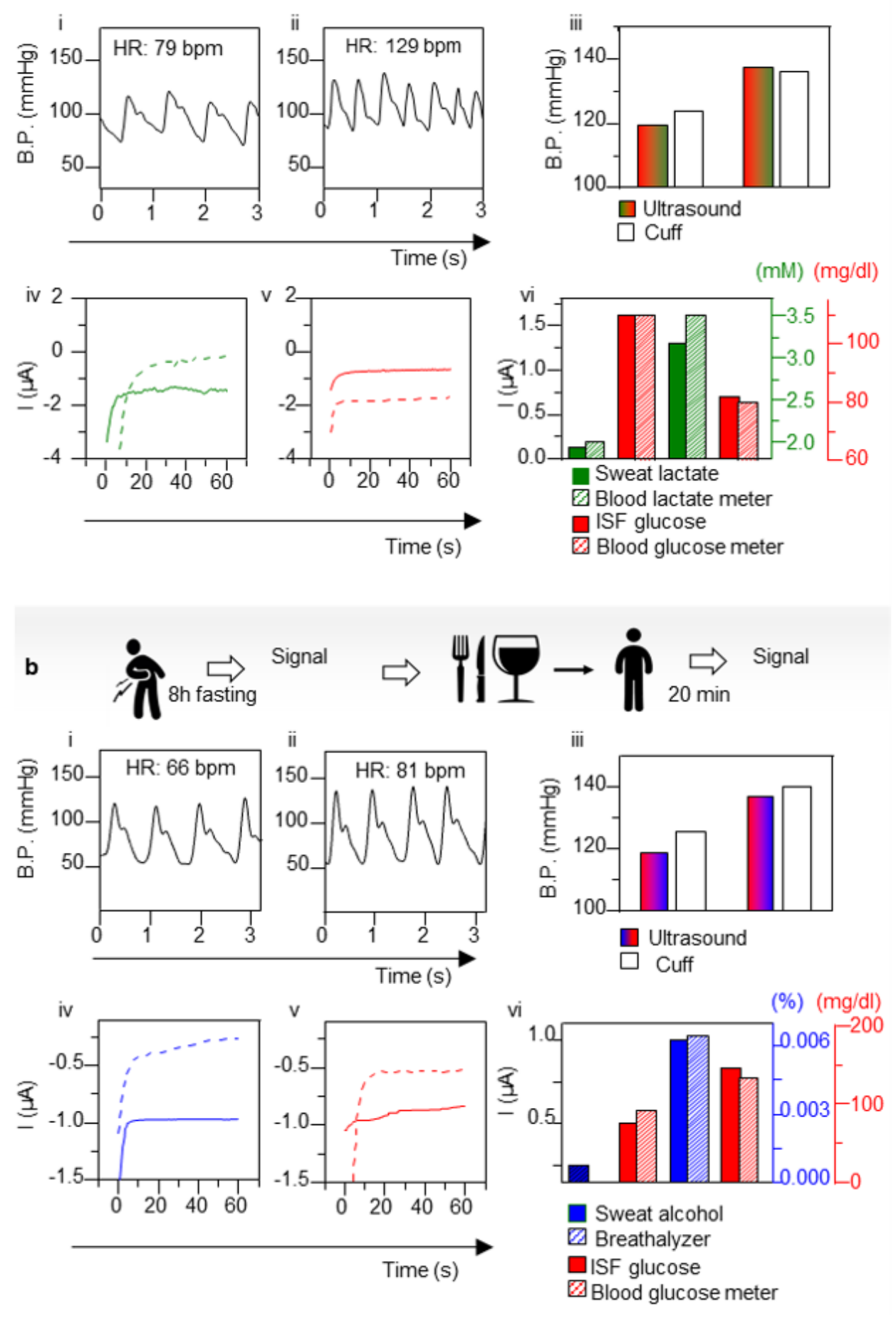

Figure 4

a, Lactate/glucose/BP performance. BP/HR signal recording before (i) and after (ii) exercise. Bar graphic comparison between BP signal using a commercial cuff (white) and the ultrasound transducer (green/red) (iii). Electrochemical sensor signal recording for sweat lactate before (dotted line) and after (solid line) exercising (iv). Electrochemical sensor signal recording for glucose after having a meal and before exercising (dotted line) and after exercise (solid line) (v). Bar graphic comparison between lactate 
levels in sweat using the electrochemical sensor (green solid) and a commercial blood lactate meter (green/white), glucose levels in ISF using the electrochemical sensor (red solid) and blood using a blood glucose meter (red/white) (vi). b. Alcohol/glucose/BP performance. BP/HR signal recording before (i) and after (ii) food and alcohol intake. Bar graphic comparison between BP signal using a commercial cuff (white) and the ultrasound transducer (blue/red) (iii). Electrochemical sensor signal recording for sweat alcohol before (dotted line) and after (solid line) alcohol intake (iv). Electrochemical sensor signal recording for ISF glucose before (dotted line) and after (solid line) food intake (v). Bar graphic comparison between alcohol levels in sweat using the electrochemical sensor (blue solid) and a commercial breathalyzer (blue/white), glucose levels in ISF using the electrochemical sensor (red solid) and blood using a blood glucose meter (red/white) (vi). 
a

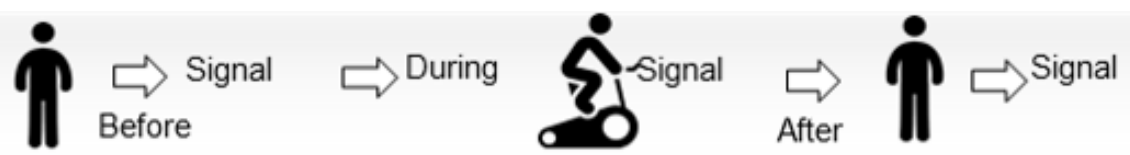
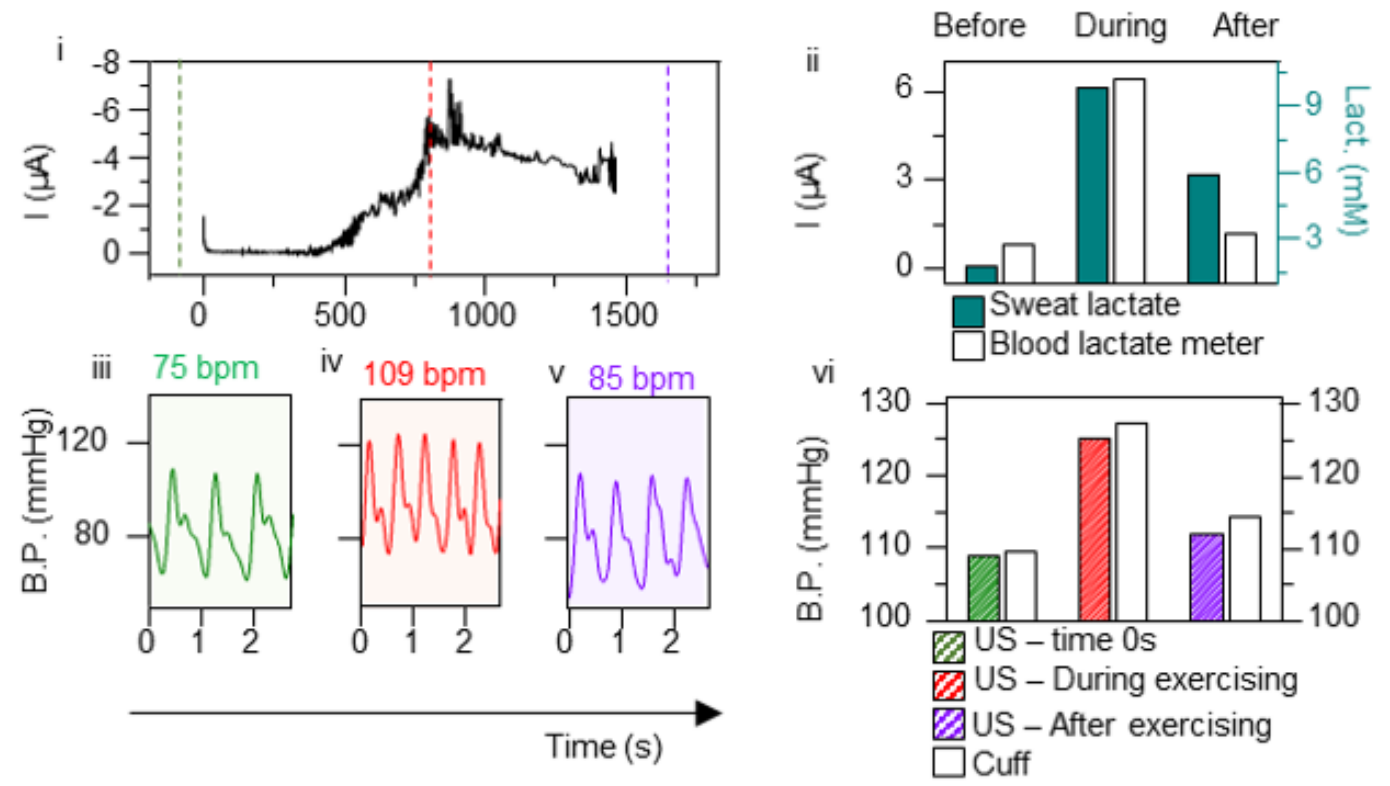

b
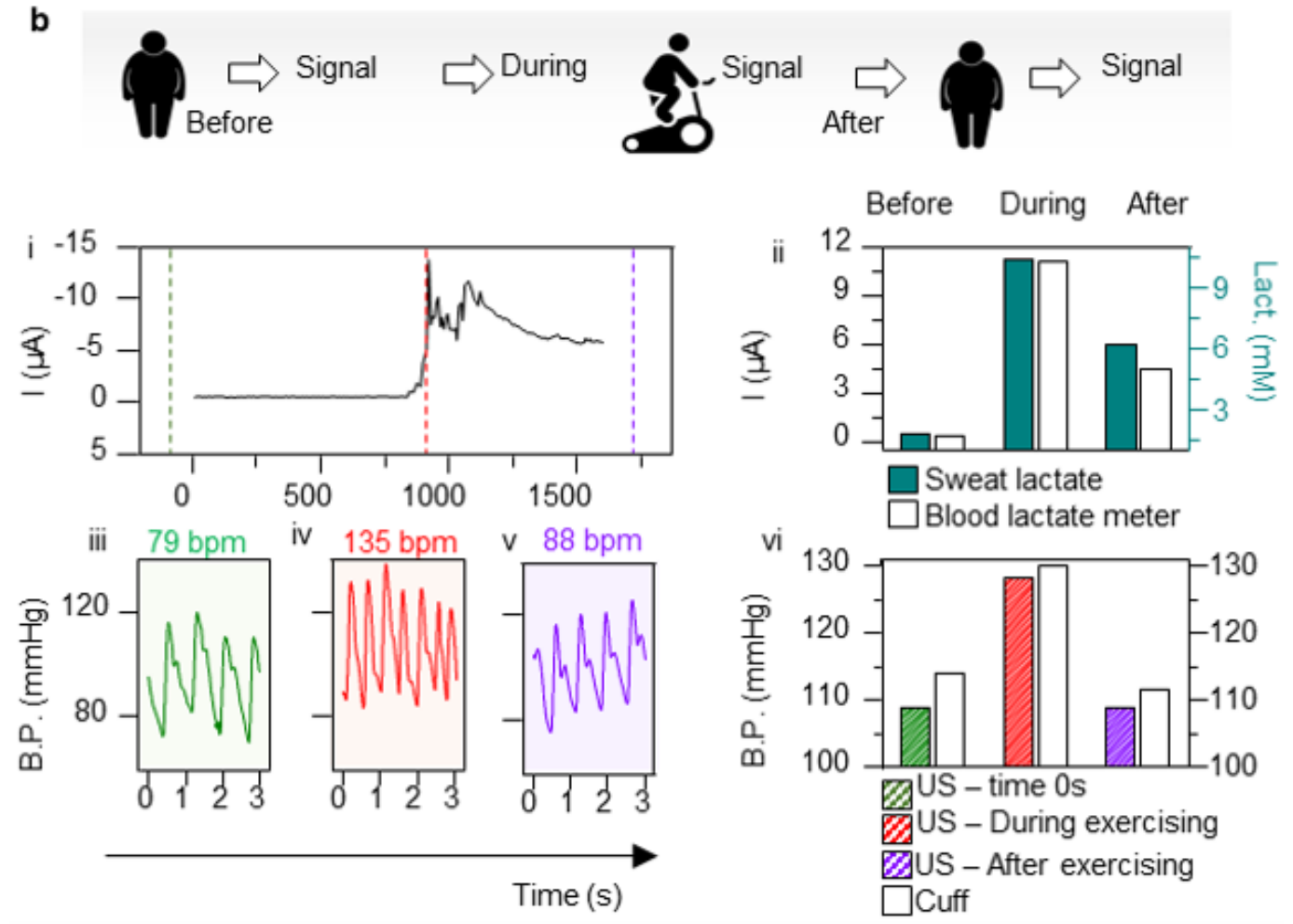

\section{Figure 5}

a, Continuous lactate/BP/HR performance for actively fit volunteer. Continuous signal recording showing sweat lactate profile during stationary biking (i). Bar graphics showing validation using a commercial blood lactate meter (white) and electrochemical sensor readings (green) (ii). BP/HR signal recording before (iii), during (iv), and after (v) stationary biking. Bar graphic comparison between BP signal using a commercial cuff (white) and the ultrasound before (green), during (red), and after (purple) of the exercise 
performance (vi). b, Continuous Lactate/BP/HR performance for sedentary volunteer. Continuous current recording showing sweat lactate profile during stationary biking (i). Bar graphics showing validation using a commercial blood lactate meter (white) and electrochemical sensor readings (green) (ii). BP/HR signal recording before (iii), during (iv), and after (v) stationary biking. Bar graphic comparison between the BP signal of a commercial cuff (white) and the ultrasound before (green), during (red), and after (purple) of the exercise activity (vi).

\section{Supplementary Files}

This is a list of supplementary files associated with this preprint. Click to download.

- SupportingInformation.docx 